\title{
Article \\ Research on Kinematic Parameters of Multiple Gait Pattern Transitions
}

\author{
Chaoyue Guo ${ }^{1,2}$, Yali Liu ${ }^{1,2, * \mathbb{D}}$, Qiuzhi Song ${ }^{1,2}$ and Shuting Liu ${ }^{3}$ \\ 1 Department of Mechanical and Engineering, Beijing Institute of Technology, 5 South Zhongguancun Street, \\ Haidian District, Beijing 100081, China; chaoyue_guo@126.com (C.G.); qzhsong@bit.edu.cn (Q.S.) \\ 2 Institute of Advanced Technology, Beijing Institute of Technology, Beijing 100081, China \\ 3 No. 14 Research Institute of the First Academy of China Aerospace Science and Technology Corporation, \\ 1 Nandahongmen Road, Fengtai District, Beijing 100076, China; liust91@gmail.com \\ * Correspondence: buaaliuyali@126.com
}

check for

updates

Citation: Guo, C.; Liu, Y.; Song, Q.; Liu, S. Research on Kinematic Parameters of Multiple Gait Pattern Transitions. Appl. Sci. 2021, 11, 6911. https://doi.org/10.3390/app11156911

Academic Editor: Augusto Ferrante

Received: 12 June 2021

Accepted: 23 July 2021

Published: 27 July 2021

Publisher's Note: MDPI stays neutral with regard to jurisdictional claims in published maps and institutional affiliations.

Copyright: (c) 2021 by the authors. Licensee MDPI, Basel, Switzerland. This article is an open access article distributed under the terms and conditions of the Creative Commons Attribution (CC BY) license (https:// creativecommons.org/licenses/by/ $4.0 /)$.
Featured Application: The current study provided more knowledge on the kinematic parameters of multiple gait pattern transition, which can be significant in designing sensor systems and gait pattern transition recognition algorithms for exoskeleton robots to cooperate with the wearer to walk and transition smoothly in a complex terrain environment and enhance people's walking ability.

Abstract: Gait recognition technology is the key technology in the field of exoskeletons. In the current research of gait recognition technology, there is less focus on the recognition of the transition between gait patterns. This study aims to determine which kinematic parameters have significant differences in the transitions (between level and stair walking and between level and ramp walking) of different gait patterns, to determine whether these parameters change differently in different gait pattern transitions, and the order the significant differences occur through a comparative analysis of various kinematic parameters between the transition stride and the before stride in the former pattern. We analyzed 18 parameters concerning both lower limbs and trunk. We compared each time point of the transition strides to the corresponding time points of the before stride using a series of two-sample $t$-tests, and we then evaluated the difference between the transition stride and the before stride based upon the number of time points within the gait cycle that were statistically different. We found that the sagittal plane angular velocity and the angular acceleration of all joints and the resultant velocity of the thigh and shank of the leading limb had significant differences in the process of transition; the sagittal plane angular velocity of all joints of the trailing limb and the velocity of the trunk in the coronary axis direction also showed a significant difference. The angular acceleration of all joints, the sagittal plane angular velocity of the ankle joint of the leading limb, and the acceleration of the trunk in the coronal axis direction showed a difference in the early stage of the transition. In general, the leading limb had a significant difference earlier than the trailing limb, and the acceleration parameters changed earlier than the velocity parameters. These parameters showed different combinations of changes in the transition of different gait patterns, and the changes in these parameters reflected different gait pattern transitions. Therefore, we believe that the results of this study can provide a reference for the gait pattern transition recognition of wearable exoskeletons.

Keywords: exoskeleton; kinematic parameters; gait pattern transition; recognition

\section{Introduction}

In the real world, we encounter complex road conditions; therefore, in the practical application of exoskeletons, we also need to take into account these conditions. Gait recognition technology is the premise that exoskeletons can cooperate with the wearer to walk and transition smoothly in a complex terrain environment and achieve a labor-saving purpose. In reality, when healthy people walk between different terrains, they adjust 
their gait strategy in time before each terrain transition in order to better maintain balance and ensure a safe and stable completion of the transition. The purpose of this study is to systematically analyze the kinematic parameters of an individual prior to the completion of a gait transition, to identify which kinematic parameters have significant differences in the transition stride when compared to the stride of a former gait pattern during a variety of gait transitions, and to determine the order the significant differences occur. This study is expected to provide a better basis for the recognition of the gait transition of the human-exoskeleton system in different terrains.

At present, the research on gait recognition in the field of exoskeletons mainly focuses on gait recognition under each terrain (pattern), including the basic research of biomechanics and the research of a recognition algorithm [1-4]. However, there are few studies on gait pattern transition, and the recognition of gait pattern transition affects the effect of human-robot cooperation. Among them, the Zhang Junxia team of Tianjin University of Science and Technology studied the biomechanical characteristics of lower limbs during stair walking, which provided the basis for the selection of exoskeleton driving elements and gait planning [5,6]. Gait transition recognition has been mentioned in some studies [7]; however, there is no systematic study and analysis of the available parameter information. Gait transition between level and stairs was studied by Martin Grimmer et al. in Germany, who analyzed whether there is a transition phase between level and stairs, the duration of the transition phase, and whether there is a joint-related order and timing for the start and end of the transitions. The study was carried out in order to provide reference for the control concept design and performance of a lower limb exoskeleton or prosthesis and to achieve the purpose of improving transition smoothness [8].

In the field of medicine, there are also many related studies that study and analyze the gait strategy of healthy individuals walking on different terrains, including the regular changes in kinematic, kinetic, and spatiotemporal parameters of the lower limbs and trunk in walking up and down stairs and slopes [9-11]. Among them, Aner Weiss et al. found that it is possible to distinguish between level walking and stair ambulation based on the kinematic parameters of the trunk [12]. In the literature [13,14], it was also pointed out that at different walking speeds the trunk movement has a corresponding change. Therefore, the kinematic and kinetic parameters of the lower limbs and trunk are of great significance for the study of gait pattern transition recognition. In addition, there have been many studies on the transitions between level and slopes, between level and stairs, and between walking and running [15-17]. Shuqi Zhang et al. found that during the transition from walking to running, the kinetic variables showed nonlinear behavior, and the joint kinetic measures actively change in nonlinear patterns before running to prepare for the gait transition. A team at Pennsylvania State University studied the spatiotemporal parameters of the transition between level and ramp, including walking speed, stride length, etc., and concluded that healthy young adults adopt a unique gait strategy different from both level and slope walking during transition strides [15]. Similarly, they analyzed the transition between level and stairs (the transition strides were defined from the left toe off on the present surface to the first left toe off on the future surface) for a greater risk of falling when walking stairs and analyzed three sagittal plane joint angles (left hip, knee, and ankle) and six muscle (biceps femoris, rectus femoris, vastus lateralis, tibialis anterior, gastrocnemius lateralis, and soleus) activity patterns, showing that the transition phases are not only an intermediate before and after the transition but also a distinct prediction of the next stride [17]. However, its definition of the transition stride is based on the movement of one leading lower limb and ignores the consideration of the movement of the other lower limb. In addition, in these studies of gait pattern transition, only the joint angle in the sagittal plane is generally involved, the joint angular velocity and the joint angular acceleration in the sagittal plane are not studied and compared, and, more importantly, the parameters related to the trunk are ignored. However, the joint angular acceleration and the joint angular velocity of these kinematic parameters relative to the joint angle will change earlier; therefore, in order to improve the recognition rate, the research of these 
kinematic parameters is very necessary. Moreover, through the study of biomechanics of human gait, we found that during the process of walking, the trunk will also swing periodically in the coronal plane [18], and, from the above-mentioned literature [12-14], we can also see that the movement of the trunk is closely related to walking speed and gait pattern. Thus, we believe that trunk-related parameters have potential applications in gait pattern transition recognition. Therefore, in addition to the study of the kinematic parameters of the leading limb (limb that enters the new gait pattern first) in the transition gait, this article also studied the kinematic parameters of the trailing limb (limb that enters the new gait pattern second) and the upper trunk, including 18 parameters (the sagittal plane angular velocity and the angular acceleration of the hip, knee, and ankle joints of both lower limbs; the resultant velocity and resultant acceleration of the thigh and shank of the leading limb; and the velocity and acceleration of the trunk in the coronal axis direction).

In short, compared with the former gait stride before the transition, we predict that there are some kinematic parameters (including the kinematic parameters of the leading limb, the trailing limb, and the trunk in the coronal plane) in the transition stride of various gait pattern transitions that show significant differences, and the differences of these parameters in different phases of the transition stride are not the same. Therefore, this study aims to redefine the transition stride and divide the transition stride into new phases in order to systematically analyze these kinematic parameters, determine which kinematic parameters have significant differences in the transitions (between level and stair walking and between level and ramp walking) of different gait patterns, to determine whether these parameters change differently in different gait pattern transitions, and the order the significant differences occur. In the future, artificial intelligence, neural networks, and machine learning algorithms can be combined to lay the foundation for exoskeleton gait pattern transition recognition research.

\section{Materials and Methods}

\subsection{Participants}

Nine healthy men (age $26 \pm 2$ years, weight $72 \pm 10 \mathrm{~kg}$, height $1.75 \pm 0.08 \mathrm{~m}$ ) completed the protocol, and all participants gave written informed consent according to the Ethics Committee of Beijing Sport University.

\subsection{Protocol}

Participants were familiarized with the procedure before the actual start of the experiment, which required the participants to start walking from one end of the experimental platform in a standing position each time and to always strike the surface of the next gait pattern with the right limb first (that is, the right limb was always the leading limb). The gait patterns are listed in Table 1. Participants completed level walking (L), stair walking (S), ramp walking (R), level walking to up-stair walking transition (L-S UP), level walking to down-stair walking transition (L-S DOWN), up-stair walking to level walking transition (S-L UP), down-stair walking to level walking transition (S-L DOWN), level walking to up-ramp walking transition (L-R UP), level walking to down-ramp walking transition (L-R DOWN), up-ramp walking to level walking transition (R-L UP), and down-ramp walking to level walking transition (R-L DOWN) at a self-selected speed on the four experimental platforms, as shown in Figure 1. Two kinds of experiments were carried out on each platform from the two ends of the platform. Each kind of experiment was a complete continuous process, including gait before transition stride, transition stride, and gait after transition stride. For example, one kind of experiment on one platform collected the data of three kinds of gait at the same time: level walking, level walking to up-stair walking transition, and up-stair walking. Similarly, another kind of experiment on this platform collected the data of down-stair walking, down-stair walking to level walking transition, and level walking at the same time. The experimental platform consisted of a footpath with a length of $4300 \mathrm{~mm}$ and a width of $1200 \mathrm{~mm}$; a platform with a length of $2000 \mathrm{~mm}$, a width of $800 \mathrm{~mm}$, and a height of $640 \mathrm{~mm}$; a $16^{\circ}$ slope with a length of $2240 \mathrm{~mm}$ and a height of 
$640 \mathrm{~mm}$ composed of multiple steps; and a four-step staircase composed of multiple steps with each step $160 \mathrm{~mm}$ in height, $800 \mathrm{~mm}$ in width, and $280 \mathrm{~mm}$ in depth. Finally, this was according to the actual experimental needs to combine the experimental platform.

Table 1. The abbreviation of gait patterns.

\begin{tabular}{ccc}
\hline No. & The Abbreviated Gait Patterns & The Meaning of Gait Patterns \\
\hline 1 & L & level walking \\
2 & S & stair walking \\
3 & R & ramp walking \\
4 & L-S UP & level walking to up-stair walking transition \\
5 & L-S DOWN & level walking to down-stair walking transition \\
6 & S-L UP & up-stair walking to level walking transition \\
7 & S-L DOWN & down-stair walking to level walking transition \\
8 & L-R UP & level walking to up-ramp walking transition \\
9 & L-R DOWN & level walking to down-ramp walking transition \\
10 & R-L UP & up-ramp walking to level walking transition \\
11 & R-L DOWN & down-ramp walking to level walking transition \\
\hline
\end{tabular}

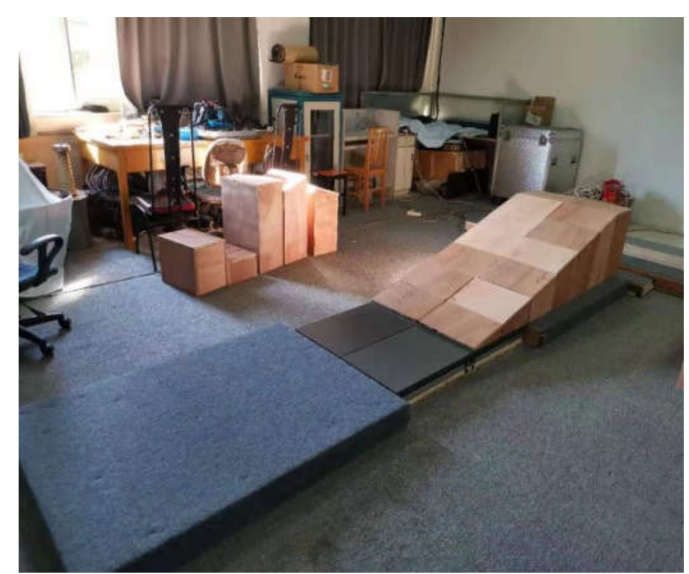

(a)

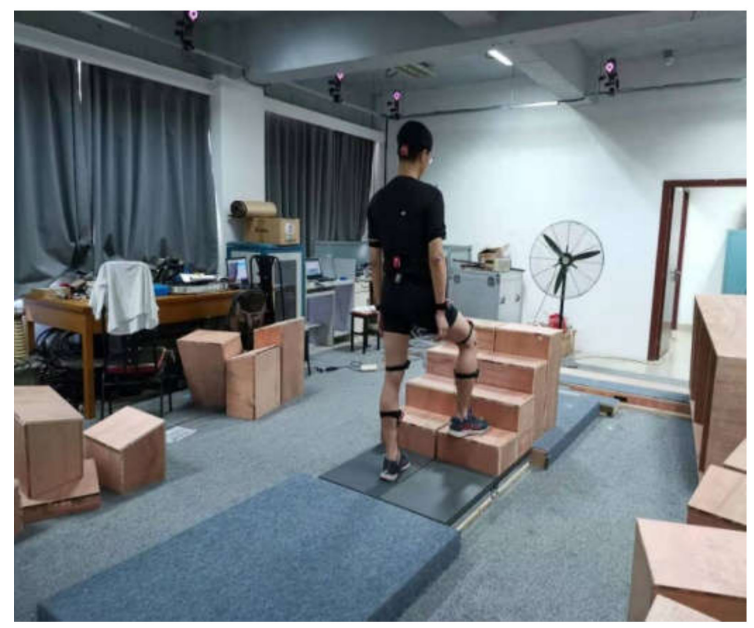

(c)

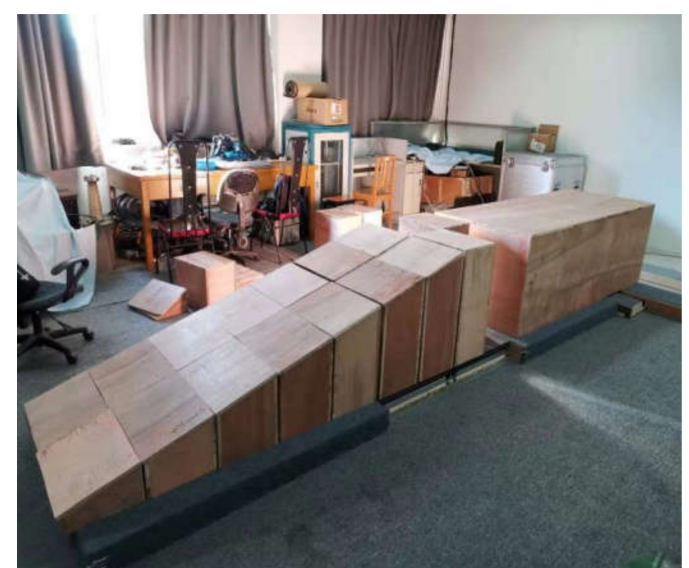

(b)

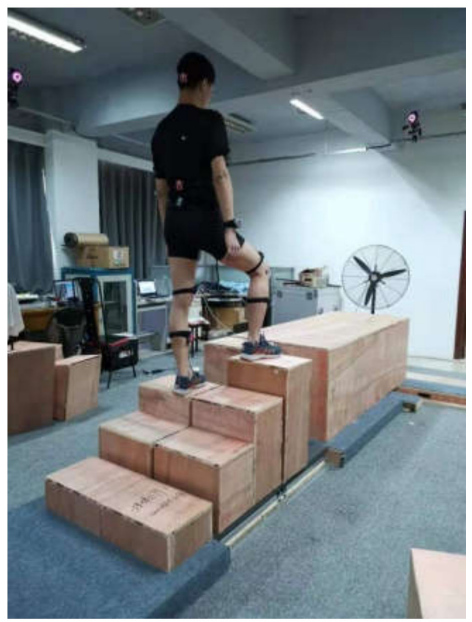

(d)

Figure 1. Experimental platform: (a) level walking to up-ramp walking and down-ramp walking to level walking; (b) upramp walking to level walking and level walking to down-ramp walking; (c) level walking to up-stair walking and down-stair walking to level walking; and (d) up-stair walking to level walking and level walking to down-stair walking.

Data were collected from five trials of participants for each kind of experiment. The complete gait pattern transition stage (transition strides) was defined from the last heel 
(toe) strike of the trailing limb in the former gait pattern to the first heel (toe) strike of the trailing limb in the next gait pattern. For example, level to up-stair transition was defined as the last heel strike of the trailing limb on the level to the first heel (toe) strike of the trailing limb on the stair. Here, due to the fact that the toe touches the surface first in the process of stair walking [19], this situation was taken into account in the transition between level walking and stair walking.

\subsection{Kinematics}

We used a 12-camera passive 3D motion analysis system (Raptor-4S) to capture the kinematic data of the participants. Biomechanical parameters of all joints and limbs except the head were collected using the Helen Hayes whole body model [20] excluding the head, and the Helen Hayes model is shown in Figure 2. The data were collected at $100 \mathrm{~Hz}$, and the data were processed by Cortex software, which included a low-pass filter at $7 \mathrm{~Hz}$ (Butterworth filter). Then, the 18 parameters (the sagittal plane angular velocity and the angular acceleration of the hip, knee, and ankle joints of both lower limbs; the resultant velocity and resultant acceleration of the thigh and shank of the leading limb; and the velocity and acceleration of the trunk in the coronal axis direction) were analyzed by SPSS software.

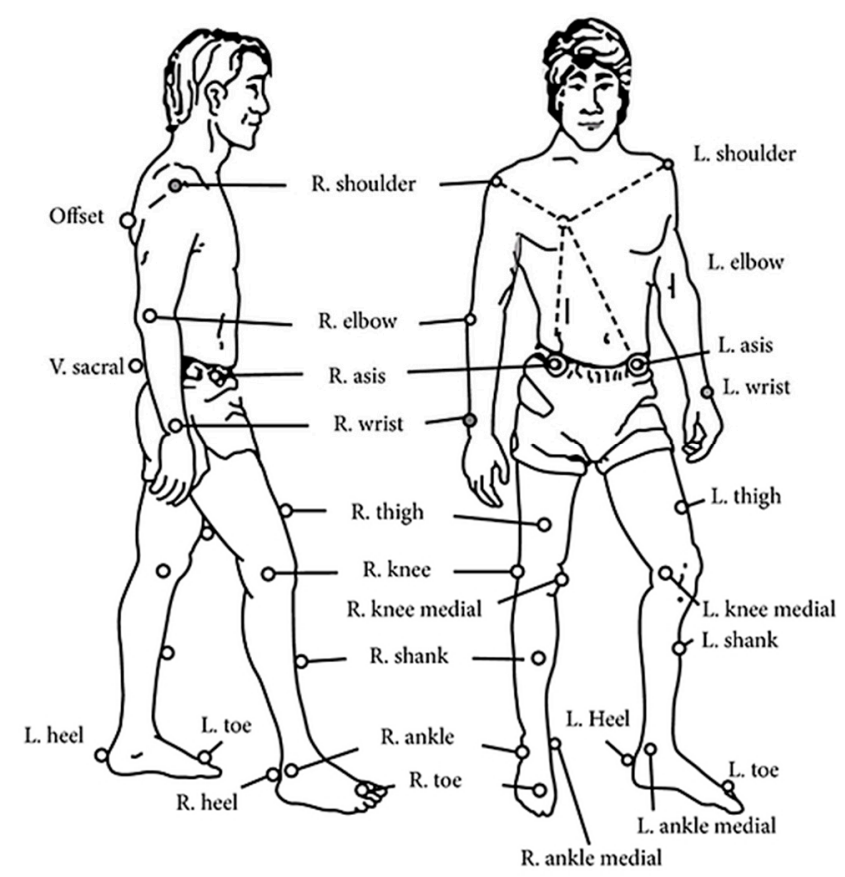

Figure 2. The Helen Hayes model.

\subsection{Data Analysis}

First, the data of each trial of experiments are intercepted, then the stride of the former pattern and the transition stride are intercepted, respectively, and the stride of the former pattern is the last complete heel strike cycle prior to the transition. To make a point-by-point data comparison, each gait cycle was resampled to 100 samples, one for each percent of the gait cycle for each gait pattern. Then, we averaged the five cycles from five trials for the 18 parameters in each condition, and then the data were normalized to the gait before transition stride by dividing each time point by the maximum value during the gait before the transition stride. We then averaged the normalized curves for each kind of experiment across all nine participants.

\subsection{Statistical Analysis}

We compared 18 parameters between the gait before transition stride and the transition stride in each experiment. For the convenience of analysis and description, the resultant 
velocity and resultant acceleration of the thigh and shank of the leading limb are called the velocity (v) and acceleration (a) of the thigh (T) and shank (S). The angular acceleration and the angular velocity in the sagittal plane of the hip, knee, and ankle joints are abbreviated as the angular acceleration (aa) and the angular velocity (av) of the hip, knee, and ankle joints. The velocity and acceleration of the trunk in the direction of the coronal axis are referred to as the velocity (v) and acceleration (a) of the trunk. First, each participant was tested at each of the 100 time points in a gait cycle in a 2-sample T-test in five trials; each T-test was for the same participant and each time point corresponded to one percent of the gait cycle. Based on the gait events of both lower limbs, the transition stride is divided into four phases. The first phase is from the heel (toe) strike of the trailing limb on the first surface to the heel (toe) off of the leading limb on the first surface. The second phase is from the heel (toe) off of the leading limb on the first surface to the heel (toe) strike of the leading limb on the second surface. The third phase is from the heel (toe) strike of the leading limb on the second surface to the heel (toe) off of the trailing limb on the first surface. The fourth phase is from the heel (toe) off of the trailing limb on the first surface to the heel (toe) strike of the trailing limb on the second surface (in the process of stair walking, the toe strike (off) first). The number of points with significant differences in each comparison and the gait phases with more significant differences were counted. Here, the parameters with more than $50 \%$ (including $50 \%$ ) of the total significant difference points were considered as the parameters with significant differences, and the parameters with more than $60 \%$ of the significant difference points concentrated in the first two gait phases were referred to as those with early significant differences. Therefore, the difference in the time and magnitude of the parameters between the transition stride and the stride before the transition stride can be measured by this method. Significance was defined as $p<0.05$.

\section{Results}

From the perspective of the whole gait cycle, almost all the acceleration parameters show no significant difference in contrast to the velocity parameters. Overall, the angular velocity (av) of the hip, knee, and ankle joints of both the leading limb and trailing limb; the velocity (v) of the thigh (T) and shank (S); and the velocity (v) of the trunk were significantly different in the comparison (Table 2). Among them, the parameters of the hip, knee, and ankle joints angular velocity with significant differences accounted for $75 \%, 69 \%$, and $63 \%$, respectively. Regarding the velocity of the thigh and the shank, the proportion of the parameters with significant differences reached $88 \%$ and $75 \%$, respectively. The velocity of the trunk was significantly different in four gait pattern transitions: level walking to up-stair walking transition (L-S UP), down-stair walking to level walking transition (S-L DOWN), level walking to up-ramp walking transition (L-R UP), and down-ramp walking to level walking transition (R-L DOWN). In particular, these parameters showed less difference in up-stair walking to level walking transition (S-L UP) and up-ramp walking to level walking transition (R-L UP). In the following, we focus on the analysis of the velocity parameters with obvious overall differences and which parameters have significant differences in the early phase, and we pay attention to the parameters with significant differences and those with significant differences in the early phase in the up-stair walking to level walking transition (S-L UP) and the up-ramp walking to level walking transition (R-L UP).

We calculated the proportion of the four gait phases in each transition gait cycle (Table 3) and then calculated the proportion of the significant difference points in each gait phase of each parameter. The results are shown in Tables 4-7. In these tables, $\mathrm{rt}$ indicates the hip of the leading limb, lt indicates the hip of the trailing limb, rs indicates the knee of the leading limb, ls indicates the knee of the trailing limb, rf indicates the ankle of the leading limb, If indicates the ankle of the trailing limb, trunk is trunk, $\mathrm{T}$ indicates the thigh of the leading limb, $\mathrm{S}$ indicates the shank of the leading limb, aa indicates angular acceleration, av indicates angular velocity, a indicates acceleration, and $\mathrm{v}$ indicates velocity. 
Table 2. Percentage difference of overall parameters (points of significant difference) (\%).

\begin{tabular}{|c|c|c|c|c|c|c|c|c|}
\hline \multirow{2}{*}{ Comparison } & \multicolumn{2}{|c|}{ L-S vs. L } & \multicolumn{2}{|c|}{ S-L vs. S } & \multicolumn{2}{|c|}{ L-R vs. L } & \multicolumn{2}{|c|}{ R-L vs. R } \\
\hline & UP & DOWN & UP & DOWN & UP & DOWN & UP & DOWN \\
\hline rt aa & 37 & 20 & 13 & 19 & 29 & 25 & 17 & 30 \\
\hline lt aa & 28 & 18 & 19 & 18 & 22 & 15 & 19 & 19 \\
\hline rs aa & 47 & 43 & 24 & 41 & 30 & 32 & 16 & 61 \\
\hline ls aa & 44 & 43 & 24 & 31 & 28 & 25 & 20 & 31 \\
\hline $\mathrm{rf}$ aa & 43 & 35 & 22 & 33 & 42 & 31 & 15 & 42 \\
\hline If aa & 42 & 29 & 19 & 33 & 40 & 28 & 16 & 31 \\
\hline rt av & 71 & 72 & 36 & 61 & 54 & 74 & 43 & 80 \\
\hline lt av & 52 & 84 & 51 & 57 & 36 & 55 & 35 & 63 \\
\hline rs av & 83 & 59 & 49 & 81 & 71 & 61 & 41 & 70 \\
\hline ls av & 70 & 81 & 43 & 59 & 66 & 32 & 32 & 71 \\
\hline $\mathrm{rf}$ av & 69 & 56 & 37 & 64 & 52 & 49 & 32 & 70 \\
\hline lf av & 48 & 51 & 36 & 52 & 56 & 56 & 20 & 48 \\
\hline $\mathrm{T} \mathrm{a}$ & 32 & 33 & 18 & 36 & 24 & 28 & 22 & 49 \\
\hline $\mathrm{Sa}$ & 37 & 43 & 19 & 42 & 28 & 34 & 20 & 60 \\
\hline $\mathrm{T} v$ & 90 & 66 & 40 & 68 & 66 & 75 & 55 & 81 \\
\hline $\mathrm{S}_{\mathrm{v}}$ & 74 & 76 & 42 & 64 & 74 & 72 & 37 & 57 \\
\hline trunk a & 34 & 30 & 29 & 37 & 25 & 28 & 13 & 19 \\
\hline trunk $\mathrm{v}$ & 56 & 30 & 36 & 56 & 52 & 31 & 29 & 79 \\
\hline
\end{tabular}

Table 3. Percentage of different gait phases over a complete gait cycle (\%).

\begin{tabular}{ccccccccc}
\hline Transition & LR UP & RL DN & LS UP & SL DN & RL UP & LR DN & SL UP & LS DN \\
\hline 1 & 28 & 19 & 17 & 21 & 20 & 18 & 17 & 18 \\
2 & 25 & 31 & 35 & 35 & 26 & 32 & 32 & 31 \\
3 & 25 & 20 & 19 & 20 & 21 & 15 & 24 & 17 \\
4 & 22 & 30 & 29 & 24 & 33 & 35 & 27 & 34 \\
\hline
\end{tabular}

Table 4. Percentage of the significant difference points in each gait phase of each parameter (\%).

\begin{tabular}{|c|c|c|c|c|c|c|c|c|}
\hline \multirow{3}{*}{ Comparison Phase } & \multicolumn{8}{|c|}{ L-S vs. L } \\
\hline & \multicolumn{4}{|c|}{ UP } & \multicolumn{4}{|c|}{ DOWN } \\
\hline & 1 & 2 & 3 & 4 & 1 & 2 & 3 & 4 \\
\hline rt aa & 19 & 32 & 31 & 18 & 21 & 49 & 8 & 22 \\
\hline lt aa & 34 & 6 & 34 & 26 & 19 & 22 & 35 & 24 \\
\hline rs aa & 19 & 43 & 23 & 15 & 22 & 30 & 8 & 40 \\
\hline ls aa & 24 & 24 & 35 & 17 & 25 & 20 & 15 & 40 \\
\hline $\mathrm{rf}$ aa & 19 & 53 & 17 & 11 & 38 & 37 & 14 & 11 \\
\hline If aa & 32 & 14 & 22 & 32 & 26 & 7 & 16 & 51 \\
\hline rt av & 13 & 41 & 23 & 23 & 22 & 30 & 11 & 37 \\
\hline lt av & 15 & 6 & 12 & 67 & 17 & 36 & 7 & 40 \\
\hline rs av & 17 & 33 & 18 & 32 & 15 & 23 & 18 & 44 \\
\hline ls av & 20 & 14 & 25 & 41 & 17 & 31 & 14 & 38 \\
\hline $\mathrm{rf}$ av & 11 & 41 & 22 & 26 & 24 & 51 & 12 & 13 \\
\hline If av & 26 & 6 & 28 & 40 & 21 & 1 & 15 & 63 \\
\hline $\mathrm{T} \mathrm{a}$ & 19 & 52 & 6 & 23 & 23 & 22 & 12 & 43 \\
\hline $\mathrm{Sa}$ & 23 & 47 & 15 & 15 & 18 & 37 & 11 & 34 \\
\hline $\mathrm{T} v$ & 18 & 33 & 20 & 29 & 28 & 26 & 2 & 44 \\
\hline $\mathrm{S}_{\mathrm{V}}$ & 19 & 35 & 22 & 24 & 22 & 30 & 13 & 35 \\
\hline trunk a & 3 & 47 & 23 & 27 & 28 & 28 & 13 & 31 \\
\hline trunk $\mathrm{v}$ & 24 & 50 & 17 & 9 & 55 & 14 & 29 & 2 \\
\hline
\end{tabular}


Table 5. Percentage of the significant difference points in each gait phase of each parameter (\%).

\begin{tabular}{ccccccccc}
\hline & \multicolumn{7}{c}{ S-L vs. S } \\
\cline { 2 - 9 } Comparison Phase & \multicolumn{7}{c}{ UP } & \multicolumn{3}{c}{ DOWN } \\
\cline { 2 - 9 } & $\mathbf{1}$ & $\mathbf{2}$ & $\mathbf{3}$ & $\mathbf{4}$ & $\mathbf{1}$ & $\mathbf{2}$ & $\mathbf{3}$ & $\mathbf{4}$ \\
\cline { 2 - 9 } & 3 & 37 & 58 & 2 & 18 & 39 & 29 & 14 \\
rt aa & 25 & 19 & 25 & 31 & 17 & 28 & 50 & 5 \\
It aa & 5 & 81 & 7 & 7 & 20 & 29 & 36 & 15 \\
rs aa & 37 & 20 & 24 & 19 & 11 & 21 & 26 & 42 \\
ls aa & 5 & 60 & 4 & 31 & 22 & 31 & 43 & 4 \\
rf aa & 7 & 13 & 24 & 56 & 36 & 30 & 21 & 13 \\
lf aa & 27 & 2 & 3 & 68 & 20 & 15 & 26 & 39 \\
rt av & 3 & 28 & 15 & 54 & 9 & 35 & 30 & 26 \\
It av & 1 & 40 & 22 & 37 & 16 & 35 & 21 & 28 \\
rs av & 18 & 4 & 12 & 66 & 29 & 24 & 25 & 22 \\
ls av & 4 & 67 & 26 & 3 & 26 & 37 & 22 & 15 \\
rf av & 18 & 3 & 11 & 68 & 23 & 10 & 26 & 41 \\
If av & 17 & 32 & 17 & 34 & 11 & 31 & 32 & 26 \\
T a & 2 & 20 & 72 & 6 & 15 & 30 & 27 & 28 \\
S a & 1 & 2 & 15 & 82 & 18 & 31 & 29 & 22 \\
T v & 3 & 32 & 15 & 50 & 6 & 33 & 32 & 29 \\
S v & 14 & 22 & 8 & 56 & 24 & 6 & 39 & 31 \\
trunk a & 30 & 2 & 11 & 57 & 21 & 17 & 28 & 34 \\
trunk v & & & & & & & &
\end{tabular}

Table 6. Percentage of the significant difference points in each gait phase of each parameter (\%).

\begin{tabular}{ccccccccc}
\hline & \multicolumn{7}{c}{ L-R vs. L } \\
Comparison Phase & \multicolumn{7}{c}{ UP } & \multicolumn{4}{c}{ DOWN } \\
\cline { 2 - 10 } & $\mathbf{1}$ & $\mathbf{2}$ & $\mathbf{3}$ & $\mathbf{4}$ & $\mathbf{1}$ & $\mathbf{2}$ & $\mathbf{3}$ & $\mathbf{4}$ \\
\cline { 2 - 9 } & 46 & 39 & 3 & 12 & 12 & 40 & 12 & 36 \\
rt aa & 37 & 8 & 12 & 43 & 13 & 27 & 13 & 47 \\
lt aa & 40 & 56 & 2 & 2 & 7 & 43 & 4 & 46 \\
rs aa & 61 & 3 & 4 & 32 & 28 & 42 & 14 & 16 \\
ls aa & 36 & 28 & 27 & 9 & 21 & 48 & 17 & 14 \\
rf aa & 50 & 4 & 10 & 36 & 21 & 33 & 6 & 40 \\
lf aa & 40 & 11 & 27 & 22 & 12 & 28 & 17 & 43 \\
rt av & 56 & 2 & 17 & 25 & 18 & 56 & 9 & 17 \\
It av & 24 & 14 & 28 & 34 & 18 & 31 & 15 & 36 \\
rs av & 45 & 18 & 12 & 25 & 33 & 12 & 16 & 39 \\
ls av & 31 & 47 & 21 & 1 & 22 & 41 & 19 & 18 \\
rf av & 41 & 3 & 27 & 29 & 18 & 27 & 20 & 35 \\
lf av & 54 & 15 & 21 & 10 & 13 & 28 & 10 & 49 \\
T a & 66 & 24 & 2 & 8 & 15 & 31 & 4 & 50 \\
S a & 16 & 7 & 40 & 37 & 22 & 38 & 18 & 22 \\
T v & 27 & 9 & 33 & 31 & 21 & 37 & 17 & 25 \\
S v & 36 & 6 & 15 & 43 & 14 & 48 & 7 & 31 \\
trunk a & 27 & 32 & 26 & 15 & 48 & 27 & 11 & 14 \\
trunk v & & & & & & & &
\end{tabular}


Table 7. Percentage of the significant difference points in each gait phase of each parameter (\%).

\begin{tabular}{ccccccccc}
\hline & \multicolumn{7}{c}{ R-L vs. $\mathbf{R}$} \\
Comparison Phase & \multicolumn{7}{c}{ UP } & \multicolumn{3}{c}{ DOWN } \\
\cline { 2 - 10 } & $\mathbf{1}$ & $\mathbf{2}$ & $\mathbf{3}$ & $\mathbf{4}$ & $\mathbf{1}$ & $\mathbf{2}$ & $\mathbf{3}$ & $\mathbf{4}$ \\
\cline { 2 - 9 } & 77 & 3 & 14 & 6 & 40 & 13 & 15 & 32 \\
rt aa & 3 & 25 & 2 & 70 & 32 & 16 & 1 & 51 \\
lt aa & 51 & 14 & 9 & 26 & 20 & 25 & 21 & 34 \\
rs aa & 31 & 4 & 13 & 52 & 21 & 3 & 23 & 53 \\
ls aa & 56 & 10 & 21 & 13 & 24 & 33 & 13 & 30 \\
rf aa & 15 & 15 & 6 & 64 & 35 & 8 & 16 & 41 \\
lf aa & 67 & 30 & 1 & 2 & 19 & 26 & 18 & 37 \\
rt av & 22 & 2 & 8 & 68 & 6 & 49 & 2 & 43 \\
It av & 34 & 4 & 8 & 54 & 23 & 39 & 18 & 20 \\
rs av & 44 & 2 & 3 & 51 & 22 & 32 & 17 & 29 \\
ls av & 34 & 38 & 23 & 5 & 24 & 37 & 16 & 23 \\
rf av & 22 & 3 & 5 & 70 & 19 & 8 & 17 & 56 \\
If av & 49 & 14 & 9 & 28 & 21 & 22 & 18 & 39 \\
T a & 81 & 5 & 1 & 13 & 21 & 32 & 13 & 34 \\
S a & 19 & 11 & 21 & 49 & 7 & 33 & 25 & 35 \\
T v & 34 & 14 & 17 & 35 & 28 & 35 & 25 & 12 \\
S v & 56 & 3 & 1 & 40 & 43 & 20 & 2 & 35 \\
trunk a & 41 & 10 & 3 & 46 & 23 & 22 & 20 & 35 \\
trunk v & & & & & & & &
\end{tabular}

\section{- $\quad$ L-S UP and L-S DOWN}

In the first two gait phases of L-S UP, all parameters of the leading limb were more differentiated than those of the trailing limb, and the points of significant difference of the velocity of the trunk were mostly concentrated in the first two phases of the gait (Table 4). The parameters with early significant differences were the angular acceleration of the knee and ankle joints of the leading limb, the acceleration of the thigh and shank, and the velocity of the trunk. However, for L-S DOWN, in the first two phases of the gait cycle, it was found that the proportion of the significant difference points was significantly increased compared with that in L-S UP (Table 4). Similarly, except for the angular velocity of the knee and ankle joints, more than half of the significant difference points of the other parameters of the leading limb were concentrated in the first two gait phases; the parameters of the trunk are also relatively concentrated in the first two phases (more than half). The parameters with early significant differences were the angular acceleration of the hip and ankle joints and the angular velocity of the ankle joint of the leading limb and the velocity of the trunk and the acceleration of the trunk (56\%).

\section{- $\quad$ S-L UP and S-L DOWN}

As mentioned above, on the whole, there are basically no parameters of significant difference in the process of ascending stairs to the level (Table 2). However, interestingly, the joint angular acceleration of the knee and ankle of the leading limb were significantly different in the second phase of S-L UP, and the significant difference points account for $81 \%$ and $60 \%$ of the transition gait cycle, respectively. In the second phase of the transition gait cycle, the angular velocity of the ankle of the leading limb was also significantly different, accounting for $67 \%$ of the gait cycle. Most of the significant difference points of the remaining parameters were concentrated in the last two phases of the gait cycle (Table 5). Therefore, for the identification of S-L UP, the parameters with early significant differences are the key reference object. During S-L DOWN, the parameters with early significant differences were the angular acceleration of the ankle of the trailing limb, the angular velocity of the ankle of the leading limb, and the angular acceleration of the leading limb hip joint (57\%). 


\section{- $\quad$ L-R UP and L-R DOWN}

During L-R UP, the parameters with early significant differences were the angular acceleration of the hip, knee, and ankle joint and the angular velocity of the ankle of the leading limb; the acceleration of the thigh (T) and shank (S); the angular velocity and angular acceleration of the knee joint of the trailing limb, as well as the angular velocity of the hip joint of the trailing limb (58\%); and the velocity of the trunk (59\%) (Table 6). During L-R DOWN, the parameters with early significant differences were the angular acceleration and velocity of the ankle of the leading limb, as well as the velocity of the thigh, the velocity of the shank (58\%), and the angular acceleration of the knee joint; the angular velocity of the hip joint in the trailing limb side; and the acceleration and velocity of the trunk (Table 6).

\section{- $\quad$ R-L UP and R-L DOWN}

During R-L UP, the parameters with early significant differences were the angular acceleration of the hip, knee, and ankle joints and the angular velocity of the ankle and hip joints of the leading limb; the acceleration of the thigh (T) and shank (S); and the acceleration of the trunk (59\%) (Table 7). Similarly, there exists the condition of fewer overall significant difference points that are instead relatively concentrated (Tables 2 and 8); the parameters with early significant differences will also provide important support for the identification of R-L UP in the future. During R-L DOWN, the parameters with early significant differences were the angular velocity of the knee and ankle joints of the leading limb as well as the velocity of the shank especially, the angular acceleration of the ankle joint of the leading limb (57\%), and the acceleration of the trunk (Table 7).

Table 8. Parameters with early significant differences in different gait pattern transitions.

\begin{tabular}{|c|c|c|c|c|c|c|c|c|}
\hline \multirow{2}{*}{ Comparison } & \multicolumn{2}{|c|}{ L-S vs. L } & \multicolumn{2}{|c|}{ S-L vs. S } & \multicolumn{2}{|c|}{ L-R vs. L } & \multicolumn{2}{|c|}{ R-L vs. R } \\
\hline & UP & DOWN & UP & DOWN & UP & DOWN & UP & DOWN \\
\hline rt aa & & $\sqrt{ }$ & & $\sqrt{ }$ & $\sqrt{ }$ & & $\sqrt{ }$ & \\
\hline \multicolumn{9}{|l|}{ lt aa } \\
\hline rs aa & $\sqrt{ }$ & & $\sqrt{ }$ & & $\sqrt{ }$ & & $\sqrt{ }$ & \\
\hline ls aa & & & & & $\sqrt{ }$ & $\sqrt{ }$ & & \\
\hline rf aa & $\sqrt{ }$ & $\sqrt{ }$ & $\sqrt{ }$ & & $\sqrt{ }$ & $\sqrt{ }$ & $\sqrt{ }$ & $\sqrt{ }$ \\
\hline If aa & & & & $\sqrt{ }$ & & & & \\
\hline rt av & & & & & & & $\sqrt{ }$ & \\
\hline lt av & & & & & $\sqrt{ }$ & $\sqrt{ }$ & & \\
\hline rs av & & & & & & & & $\sqrt{ }$ \\
\hline ls av & & & & & $\sqrt{ }$ & & & \\
\hline rf av & & $\sqrt{ }$ & $\sqrt{ }$ & $\sqrt{ }$ & $\sqrt{ }$ & $\sqrt{ }$ & $\sqrt{ }$ & $\sqrt{ }$ \\
\hline \multicolumn{9}{|l|}{ If av } \\
\hline $\mathrm{T} \mathrm{a}$ & $\sqrt{ }$ & & & & $\sqrt{ }$ & & $\sqrt{ }$ & \\
\hline $\mathrm{S} \mathrm{a}$ & $\sqrt{ }$ & & & & $\sqrt{ }$ & & $\sqrt{ }$ & \\
\hline $\mathrm{T} v$ & & & & & & $\sqrt{ }$ & & \\
\hline $\mathrm{S} v$ & & & & & & $\sqrt{ }$ & & $\sqrt{ }$ \\
\hline trunk a & & $\sqrt{ }$ & & & & $\sqrt{ }$ & $\sqrt{ }$ & $\sqrt{ }$ \\
\hline trunk v & $\sqrt{ }$ & $\sqrt{ }$ & & & $\sqrt{ }$ & $\sqrt{ }$ & & \\
\hline
\end{tabular}

$\sqrt{ }$ in the table indicates that the corresponding parameters have significant differences earlier in the comparison, and the red $\sqrt{ }$ indicates that the corresponding parameters which have significant difference points in the first two phases are between $55 \%$ and $60 \%$.

From the analysis of the parameter differences in the four phases of the complete gait cycle, it can be seen in Table 8 that the parameters that show significant differences earlier in the different gait pattern transitions are also different. We found that the angular acceleration of each joint changes earlier than the angular velocity, except for the ankle joint of the leading limb. Relatively speaking, in the eight kinds of gait transition, the parameters that appeared to have early significant differences were mostly the parameters of the leading limb, including the angular acceleration of the hip, knee, and ankle joints and the angular velocity of the ankle of the leading limb. The angular acceleration and angular velocity of 
the ankle of the leading limb appeared to have significant differences in the first two phases of all eight kinds of transition stride. In addition, for S-L UP and R-L UP, these parameters showed less difference in overall gait cycle; however, the significant differences of some parameters were relatively concentrated in the first two phases of the gait cycle.

In general, the angular velocity of the hip, knee, and ankle joints of the leading limb and trailing limb; the velocity of the thigh and shank; and the velocity of the trunk were significantly different in the comparison of transition strides and the strides of the former gait pattern. However, these parameters showed less difference in up-stair walking to level walking transition (S-L UP) and up-ramp walking to level walking transition (R-L UP) (essentially all parameters differ by less than $50 \%$, and those that differ by between $40 \%$ and $50 \%$ differ by less than $30 \%$ ). Among them, the velocity of the trunk was significantly different in four gait pattern transitions: level walking to up-stair walking transition (L-S UP), down-stair walking to level walking transition (S-L DOWN), level walking to upramp walking transition (L-R UP), and down-ramp walking to level walking transition (R-L DOWN) (Table 2). However, the acceleration parameters that showed no significant difference in the whole gait cycle, including the angular acceleration of each joint of the leading limb, the acceleration of the thigh and shank, and the acceleration of the trunk, showed more significant differences in the early phases. Moreover, their differences in the different gait pattern transitions were also different.

\section{Discussion}

In general, as we expected, our results show that there are some unique gait strategies in the transition of multiple gait patterns, and there are some kinematic parameters in the transition, including the kinematic parameters of the leading limb and the trailing limb, and the kinematic parameters of the trunk in the coronal plane intuitively show the difference between the transition stride and the stride of the former gait pattern. Moreover, some parameters were adjusted and changed actively before the leading limb struck the surface of the next gait pattern. However, the parameters that have significant differences in different gait patterns are not the same, and the order that the differences occur is not the same. This is partly confirmed by the study of Martin Grimmer et al., who found that during the transitions between level and stair, each joint of the lower extremity (knee, hip, and ankle) exists in both transition phases, and each joint has a different order of change in the transition between level and stair, both up and down [8]. Therefore, in the application of exoskeleton gait recognition, we can identify the changes in these kinematic parameters to achieve the recognition of a variety of gait pattern transitions and even predict them in advance so that the human-exoskeleton system can timely and accurately adjust the control mode to achieve a smooth transition.

- $\quad$ Leading limb (right)

Overall, all relevant kinematic parameters, except the angular acceleration and the acceleration of the thigh and shank, showed significant differences in multiple transitions except for S-L UP and R-L UP (Table 2). For S-L UP, it may be because compared to the stair ascent stride, the swing trajectory of the leading limb in the transition stride between the up-stair walking and level walking basically does not change much before the leading limb strikes the surface of level (both lift two steps), and the difference mainly focuses on the difference of the trailing limb in the last two phases of the transition stride (the trailing limb only needs to lift to a one-step height) (Figure 3a); this is consistent with the work of Riley C. Sheehan et al. [17]. For R-L UP, the swing trajectory of the leading limb changes a little (Figure 3b), which is also because the height that the leading limb needs to lift to is of little difference. However, the differences of the angular acceleration of the hip, knee, and ankle of the leading limb are mostly concentrated in the first two phases in the process of S-L UP and R-L UP. Therefore, in the future, we need to focus on the angular acceleration of the hip, knee, and ankle of the leading limb for the recognition of these two kinds of gait pattern transition. 

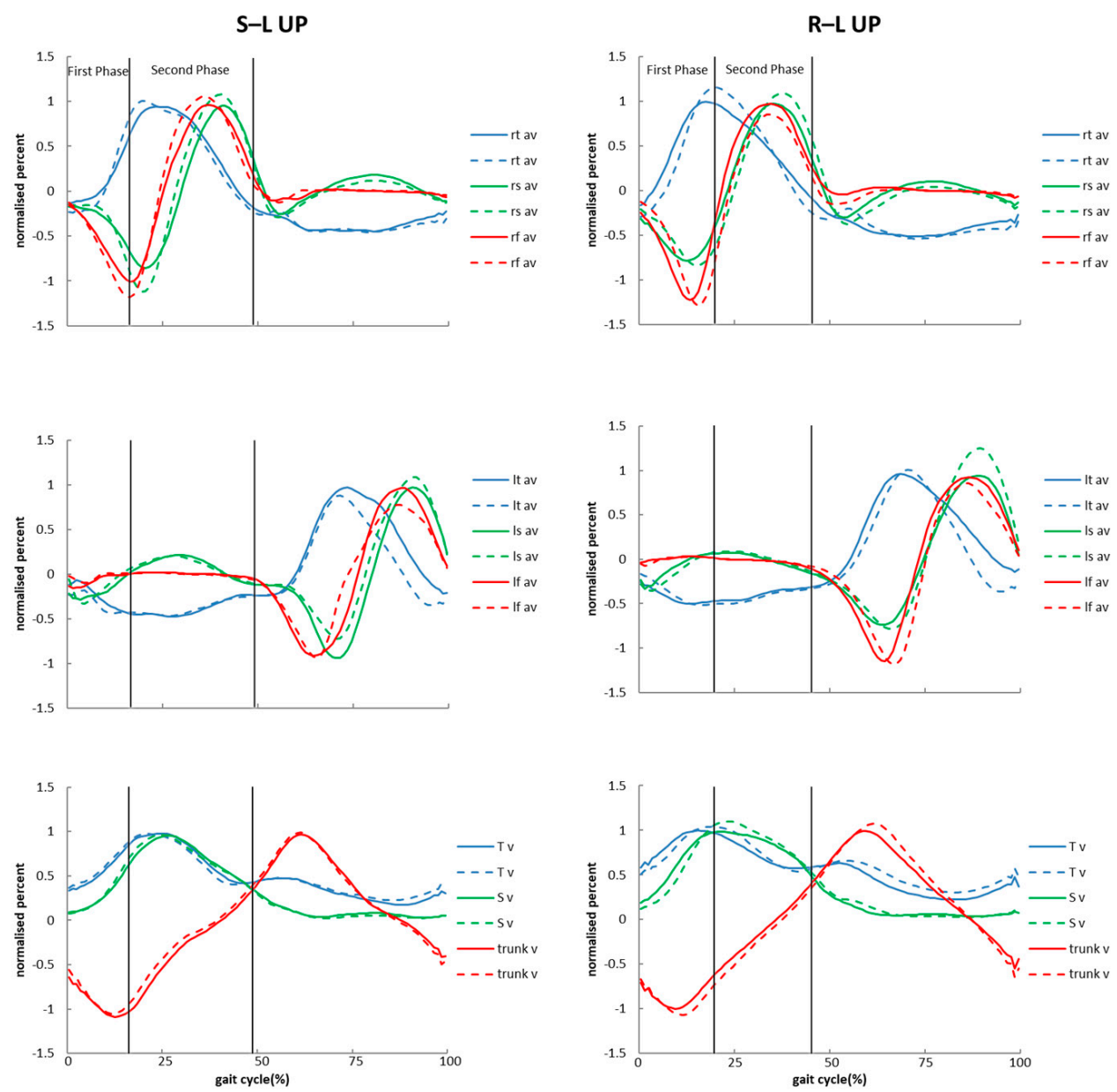

(a)

(b)

Figure 3. Average profile of the parameters: (a) during S-L UP and (b) during R-L UP. rt indicates the hip of leading limb, 1t indicates the hip of trailing limb, rs indicates the knee of leading limb, ls indicates the knee of trailing limb, rf indicates the ankle of leading limb, If indicates the ankle of trailing limb, trunk is trunk, T indicates the thigh of the leading limb, $\mathrm{S}$ indicates the shank of the leading limb, aa indicates angular acceleration, av indicates angular velocity, a indicates acceleration, and $\mathrm{v}$ indicates velocity. The dashed lines indicate transition stride. The solid lines indicate the stride before transition stride.

During S-L DOWN and R-L DOWN, the kinematic parameters of the leading limb were significantly different. First, the angular velocity of the hip, knee, and ankle joint of the leading limb and the velocity of the thigh and shank were the significant difference parameters during S-L DOWN. In the first two phases of the gait cycle, the angular velocity of the ankle joint is significantly different (Table 8), while the angular velocity of the hip and knee joints has a larger amplitude of variation (Figure 4a). On the whole, these parameters are faster in S-L DOWN than in down-stair walking, and that is possible when people pass through a complex terrain and their gaze fixation is highly related to the terrain they are about to step on [21].Moreover, from a previous study [22], it can be seen that the peak value of the angular velocity will increase with the increase in walking speed; thus, the parameters will change as described above in order to reach the relatively safer level ground faster in the process of S-L DOWN. During R-L DOWN, as can be seen from Figure $4 \mathrm{~b}$, in the first two phases, the angular velocity of the hip, knee, and ankle joints of the leading limb in the transition stride is obviously delayed compared with that in the down-ramp walking stride, and the phase difference is normal: previous studies have shown that the 
percentage of gait phase varies with pace and also that the duration of the support phase and swing phase will change [23,24]; however, in the study of Jinger S. Gottschall et al., it was found that due to the effect of gravity on the slope, both the pace and the step length were increased during R-L DOWN when compared with level walking. Moreover, the pace and the step length in the down-ramp walking are smaller than those in the level walking [15], which is also supported by the research of Jin-Tae Han et al. [9]; therefore, the pace and the step length during R-L DOWN are improved relative to down-ramp walking. It is thus inferred from [22] that the angular velocities in R-L DOWN should be faster than the angular velocities in the down-ramp walking. We can see from Figure $4 \mathrm{~b}$ that the angular velocities of each joint change from slower to faster in the transition stride than in the down-ramp walking. In addition, it can also be seen from Figure 4 that in the second phase of the gait cycle, the velocity of the thigh and shank in transition strides is faster than that in both the down-ramp walking and down-stair walking, and the variation amplitude in the down-ramp walking is larger than that in the down-stair walking. The reason for this, in addition to the common factor of reaching the safety interface faster, is that between the slope and the horizontal plane we have a certain inclination, which can vary in the propulsion strategy of the trailing limb and the effect of gravity.
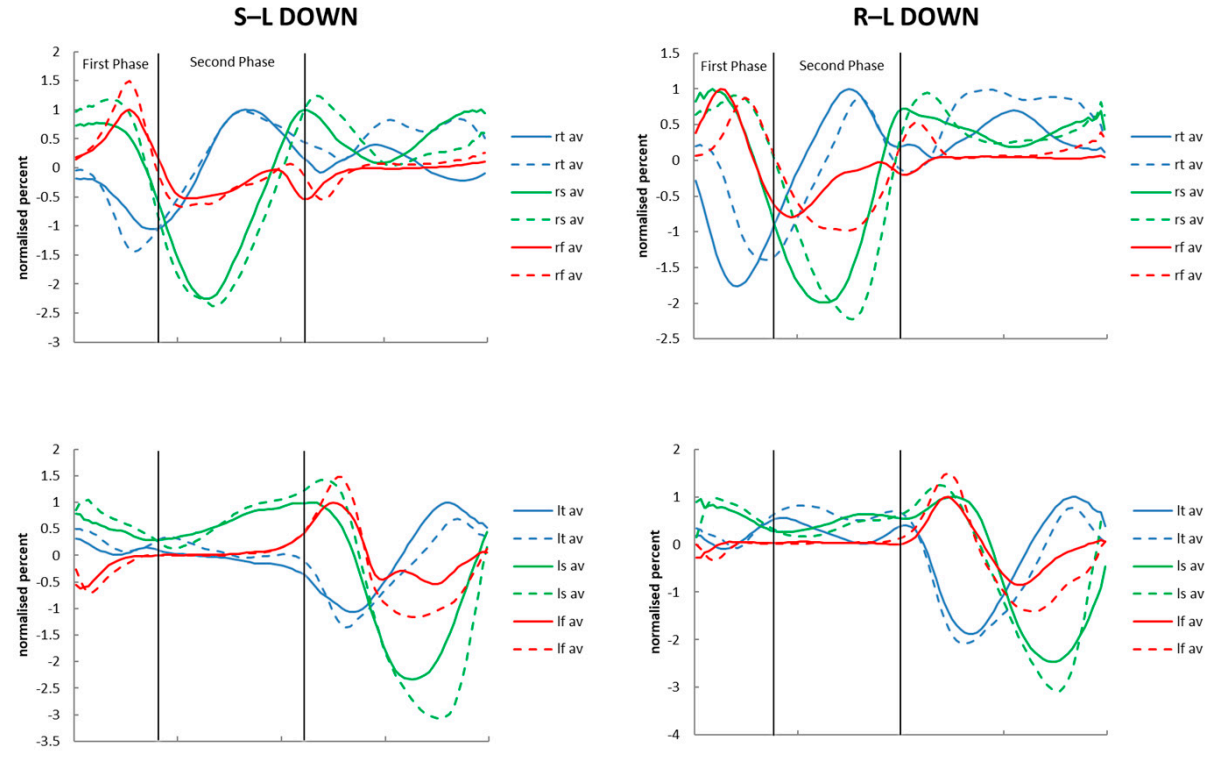

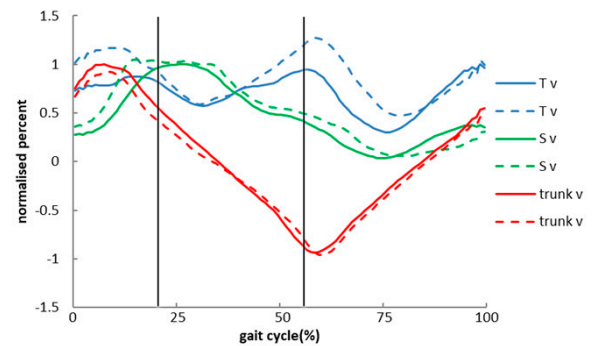

(a)

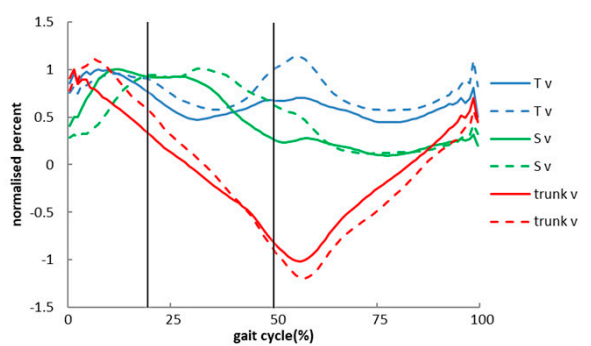

(b)

Figure 4. Average profile of the parameters: (a) during S-L DOWN and (b) during R-L DOWN. rt indicates the hip of leading limb, lt indicates the hip of trailing limb, rs indicates the knee of leading limb, ls indicates the knee of trailing limb, rf indicates the ankle of leading limb, lf indicates the ankle of trailing limb, trunk is trunk, $\mathrm{T}$ indicates the thigh of the leading limb, $\mathrm{S}$ indicates the shank of the leading limb, aa indicates angular acceleration, av indicates angular velocity, a indicates acceleration, and $\mathrm{v}$ indicates velocity. The dashed lines indicate transition stride. The solid lines indicate the stride before transition stride. 
During L-S DOWN, the angular velocity of the hip, knee, and ankle joints of the leading limb and the velocity of the thigh and shank in the transition stride were lower than that in level walking in most phases of the gait cycle, and the phase of the transition stride was slightly advanced relative to the level walking (Figure 5a). In the whole gait cycle, although the parameters with early significant differences of the leading limb accounted for a small proportion (Table 8), the overall number of significant difference points of these parameters of the leading limb is still large, and the parameter change range is large (Figure 5a). Similarly, the angular velocity of each joint of the leading limb and the velocity of the thigh and shank are mostly lower than that of level walking during L-R DOWN (Figure 5b). The parameters with early significant differences of the leading limb during L-R DOWN were the angular velocity of the ankle joint and the velocity of the thigh and shank (Table 8). For L-S DOWN see Figure 5a, and for L-R DOWN see Figure 5b. In order to cross the transition boundary more safely, the pace and step length in transition strides will be reduced relative to the level walking to ensure a balanced and stable transition. Joshua Peng et al. found that people's walking speed decreased when they transitioned from level walking to stair walking [25]. Moreover, changes in pace and step length are accompanied by corresponding changes in phase and joint angular velocity [22-24].

During L-S UP (Figure 6a) and L-R UP (Figure 6b), previous studies have revealed some differences in the transitions. Prentice SD et al. studied the changes in limb trajectory and body posture when people transitioned from level to slopes with different gradients, and they found that during the transition from level to ramp, the pitch of the trunk in the sagittal plane and the swing limb changed correspondingly at the beginning and end of the swing [25]. In order to cross the obstacles (stairs) more safely during the transition, the pace and the step length will be reduced [26], and the foot clearance (FC) will be increased [27] compared with that of the level walking, which are the main reasons for the increase in the significant differences of kinematic parameters. In the comparison during L-S UP, we found in the first two phases that the angular velocity of the hip, knee, and ankle joints of the leading limb and the velocity of the thigh and shank changed from faster to slower than that in level walking (Figure 6a). Similarly, the angular velocity of the hip and knee joints of the leading limb during L-R UP also had the same phenomenon (Figure 6b). However, the change in these parameters of the leading limb during L-R UP is not so obvious compared with that during L-S UP. We analyzed the reasons for this phenomenon and found that in L-S UP and L-R UP, people will quickly lift their limb and dorsiflex foot to prevent tripping over obstacles (stairs, ramp); this is a special kind of gait strategy (to keep balance, quickly lift lower limb to prevent stumbling at the same time).

According to our statistical results, although the total significant difference points of the angular acceleration of each joint of the leading limb in different gait pattern transitions are not many, in the first two phases of most transition strides, the angular acceleration of the hip, knee, and ankle joints showed significant differences earlier. Therefore, from the practical application point of view, the angular acceleration of each joint of the leading limb can be considered as the reference parameters of the exoskeleton gait recognition.

- Trailing limb (left)

According to our literature survey, there are few studies on the kinematic parameters of the trailing limb in the transition stride. Therefore, this paper also makes a comparative study of the kinematic parameters of the trailing limb between the transition stride and the stride of the former gait pattern during multiple gait pattern transitions in order to provide a more comprehensive data support for the subsequent feature selection and extraction. Firstly, same as the leading limb, the angular velocity of each joint of the trailing limb is significantly different in most gait pattern transition comparisons except for the comparisons of S-L UP and R-L UP (Table 2, as can be seen from the comparison of Figures 3-6). Specifically, the angular velocity of the hip joint of the trailing limb showed a significant difference during S-L UP (Table 2, 51\% significant difference points), and the angular velocity of the hip joint in the transition stride is lower than that in up-stair walking (Figure 3a). Therefore, from this point of view, the angular velocity of the hip joint of the 
trailing limb is a parameter to be focused on when recognizing S-L UP. Moreover, generally, from the distribution of the significant difference points of the trailing limb in each phase of the gait cycle, we can clearly see that in all the gait patterns transition comparisons, the differences of basically all the parameters mostly occur in the last two phases of the gait cycle (Tables 4-8).
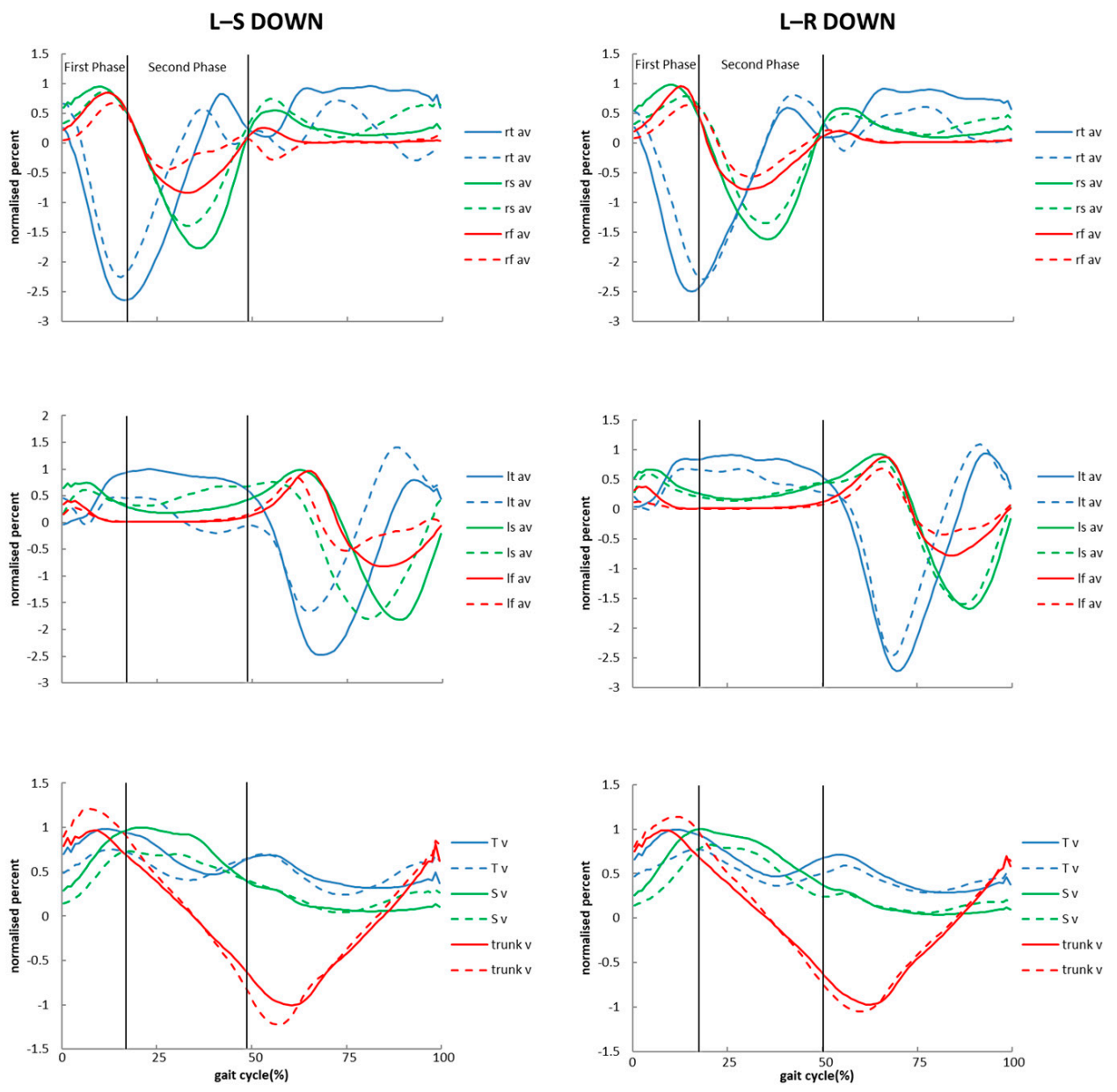

(a)

(b)

Figure 5. Average profile of the parameters: (a) during L-S DOWN and (b) during L-R DOWN. rt indicates the hip of leading limb, It indicates the hip of trailing limb, rs indicates the knee of leading limb, ls indicates the knee of trailing limb, rf indicates the ankle of leading limb, lf indicates the ankle of trailing limb, trunk is trunk, $\mathrm{T}$ indicates the thigh of the leading limb, $\mathrm{S}$ indicates the shank of the leading limb, aa indicates angular acceleration, av indicates angular velocity, a indicates acceleration, and $\mathrm{v}$ indicates velocity. The dashed lines indicate transition stride. The solid lines indicate the stride before transition stride. 

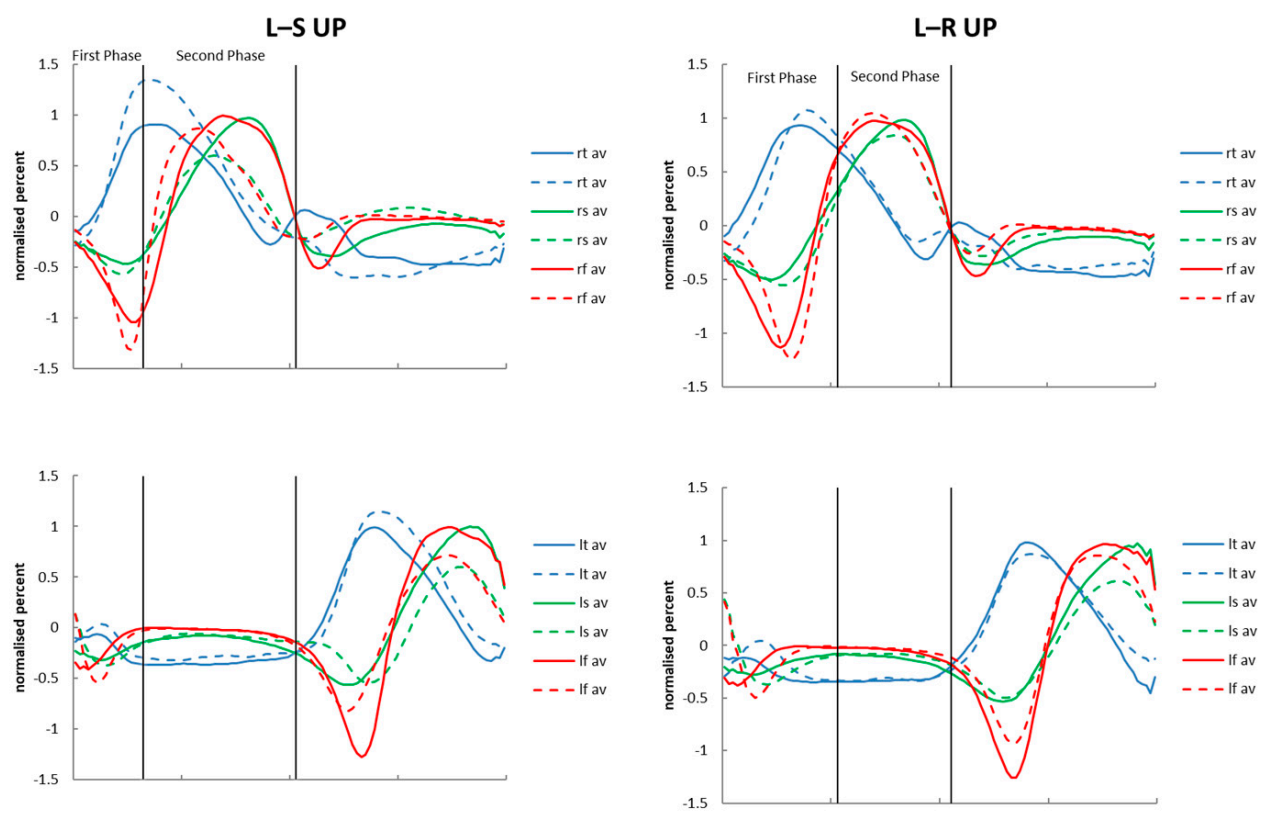

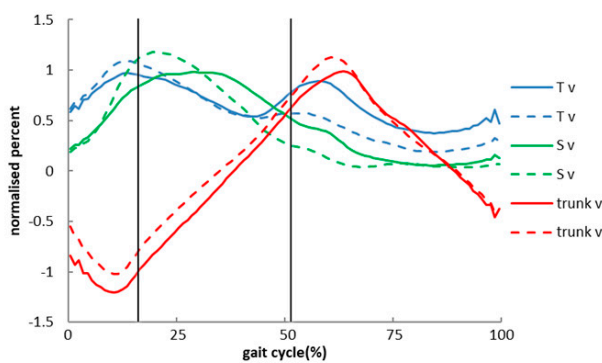

(a)

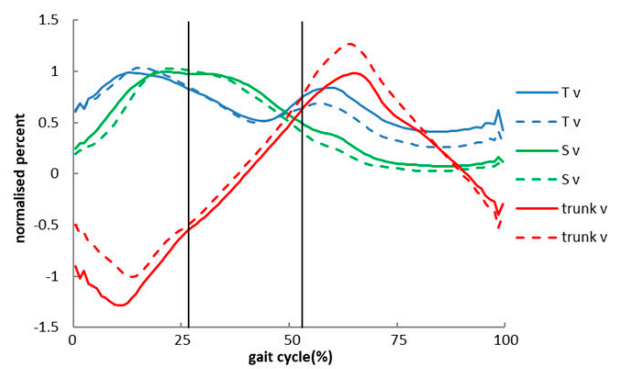

(b)

Figure 6. Average profile of the parameters: (a) during L-S UP and (b) during L-R UP. rt indicates the hip of leading limb, lt indicates the hip of trailing limb, rs indicates the knee of leading limb, ls indicates the knee of trailing limb, rf indicates the ankle of leading limb, If indicates the ankle of trailing limb, trunk is trunk, T indicates the thigh of the leading limb, $\mathrm{S}$ indicates the shank of the leading limb, aa indicates angular acceleration, av indicates angular velocity, a indicates acceleration, and $\mathrm{v}$ indicates velocity. The dashed lines indicate transition stride. The solid lines indicate the stride before transition stride.

We also found two special cases in the process of analyzing the data of the trailing limb. One is that the total significant difference points are few, and the difference points are relatively concentrated in the first two phases of the gait cycle. The other is that there are more total significant difference points, and the proportion of the difference points in the first two phases of the gait cycle is close, but it does not reach $60 \%$. We believe that the impact of these two types of conditions on gait pattern transition recognition cannot be ignored. Category one: from Table 8, it can be seen that the angular velocity of the hip joint of the trailing limb is a parameter with early significant difference for L-R UP, but the significant difference points of the angular velocity of the hip joint in the whole cycle are less, only $36 \%$ (Table 2); moreover, the angular velocity of the knee joint of the trailing limb in the L-R DOWN stride showed a difference earlier compared with level walking (Table 8), the number of points of significant difference during the whole cycle was only $32 \%$ (Table 2). Category two: during L-R DOWN, the significant difference points in the first two phases of the angular velocity of the trailing limb hip joint accounted for $49 \%$ (Table 6), but there were more significant difference points in the whole gait cycle, and the angular velocity of the hip joint in the transition stride was greatly reduced compared with 
that in the level walking (Figure 5b). Thirdly, during R-L DOWN, the angular velocity of the hip and knee of the trailing limb in the first two phases of the gait cycle had a great change in the transition stride compared with that in the down-ramp walking. In the second phase of the gait cycle, the angular velocity of the knee was lower in the transition stride than that in the down-ramp walking (Figure $4 \mathrm{~b}$ ) in contrast to the angular velocity of the hip. Both of the two proportions of difference points in the first two phases of the gait cycle were less than $60 \%$. In the process of L-S DOWN, the angular velocity of the hip and knee joint of the trailing limb also showed a similar condition (Table 4), but these two parameters had more than $50 \%$ significant difference points over the entire gait cycle, and both of them varied greatly (Figure 5a). However, the difference between these two types of parameters can be exploited by the research of a recognition algorithm. For the above two cases, we have reason to use these relevant parameters as reference parameters in future gait recognition applications. Therefore, from a comprehensive point of view, the angular velocity of each joint of the trailing limb can be considered as a reference parameter for gait pattern recognition, and the angular velocity of the hip and knee joint can be focused on to improve the rate of gait pattern recognition. Overall, in terms of the angular acceleration of the trailing limb joints, there was no significant difference in any comparison of gait pattern transitions on the whole (Table 2) nor was there a feature of early difference occurring. Therefore, in future research of gait recognition algorithms, the angular acceleration of each joint of the trailing limb will not be taken as a reference object.

- Trunk

The velocity of the trunk in the direction of the coronal axis was significantly different in the comparison of S-L DOWN (Figure 4a), R-L DOWN (Figure 4b), L-S UP (Figure 6a), and L-R UP (Figure 6b) (Table 2). Moreover, the velocity of the trunk is a parameter with early significant difference for L-R UP and L-S UP (Table 8). We can also see that in the first two phases of the gait cycle during L-R UP and L-S UP, the velocity of the trunk in the transition strides is lower than that in level walking (Figure 6), and this change is in accordance with the gait strategy that in order to more safely over obstacles (stairs, slopes), people in the transition process will reduce the velocity of the trunk to keep a better balance and then raise the lower limbs quickly to avoid tripping over. In addition, in the first two gait phases of the gait cycle, the velocity of the trunk in the transition stride is lower than that in down-stair walking during S-L DOWN, but the variation was not significant (Figure 4a). In contrast, during R-L DOWN, the velocity of the trunk in the transition stride is higher than that in down-ramp walking (Figure $4 \mathrm{~b}$ ). In addition, the overall difference between L-R DOWN and L-S DOWN is not much. However, from Table 8 and Figure 5, we can see that the velocity of the trunk in the first two phases of the gait cycle is significantly different; it is higher in both transition strides than that in level walking, and the range of change is large. Another kinematic parameter of the trunk, the acceleration, does not show significant differences throughout the gait cycle (Table 2) but shows differences earlier in the gait cycles of multiple gait pattern transitions (Table 8). Therefore, in general, the velocity of the trunk can be used as the main reference parameter in gait recognition, and the swing acceleration of the trunk can be used as an auxiliary reference.

This paper focuses on the study of kinematic parameters of the transition stride during gait pattern transitions, mainly considering that in the practical application of exoskeleton gait recognition, kinematic parameters can be obtained more conveniently and directly, and the cost of the sensor system to obtain these parameters is also lower. However, our analytical methods and experimental devices still have some limitations. The main limitation of the analytical method is that the $\alpha$ level is 0.05 , which means that for every $100 t$-tests we perform, there may be five false positives; this leads to a large number of $t$-tests being more prone to false positives. However, in order to reduce the influence of this problem, we define the parameter with as many significant difference points as possible as a significant difference parameter so that the number of possible false positive points relative to the total number of points can be ignored. Moreover, during our analysis of the data, we found that at the alpha level of 0.01 , there were also relatively more significant 
difference parameters. Furthermore, there are limitations to experimental devices such as stairs and ramps. First, the stairs have only four steps, which may not enable a person to achieve a stable stair-walking gait during walking. In addition, Riley C. Sheehan et al. found that different slopes have different transition strategies [28]. In this paper, only the transition between the level and the ramp with one slope was studied. In addition, the experimental data were resampled; it is almost impossible to perfectly line up the data when comparing parameter data. However, in this paper, through the evaluation criteria to select the more significant difference parameters, is a feature selection that, to some extent, can select the difference in relatively more significant parameters.

In the future, we hope to perform a similar analysis on the transition of walking on more inclined slopes and more complex terrains and make more detailed comparisons for the different transitions among these terrains directly so as to understand the parameters with significant differences in general applicability in human gait pattern transition, which will be helpful for the construction and optimization of exoskeleton sensor systems and the improvement of gait recognition algorithms in terms of rapidity and accuracy.

\section{Conclusions}

To sum up, the results of this study show that there are different gait strategies in the transition of multiple gait patterns, which are reflected in the kinematic parameters of the leading limb, the trailing limb, and the trunk: as a whole, the angular velocities in the sagittal plane of each joint, the resultant velocities of the thigh and shank of the leading limb, the angular velocities in the sagittal plane of the trailing limb, and the velocity of the trunk in the coronal axis direction were significantly different in the adjustment of gait pattern transition. As far as the time sequence is concerned, the joint angular acceleration of the leading limb, the angular velocity of the ankle joint in the sagittal plane, and the acceleration of the trunk in the coronal axis direction show significant differences earlier in the early stage of the transition stride. Usually, the leading limb changes significantly before the trailing limb, and the acceleration parameters change significantly before the velocity parameters.

In brief, these parameters show different combinations of changes in each gait pattern transition, and the changes in these parameters reflect different gait pattern transitions. Therefore, in future research of exoskeleton gait recognition, the fusion of this parameter information can be used as a support and combined with artificial intelligence and machine learning algorithms to improve the accuracy and rapidity of gait recognition.

Author Contributions: Conceptualization, C.G. and Q.S.; methodology, C.G.; investigation, C.G.; resources, C.G., Q.S. and Y.L.; data curation, C.G.; writing—original draft preparation, C.G.; writingreview and editing, C.G., Y.L. and S.L.; visualization, C.G.; supervision, Q.S.; project administration, Q.S. and Y.L.; funding acquisition, Q.S. and Y.L. All authors have read and agreed to the published version of the manuscript.

Funding: This research was funded by grants from the National Natural Science Foundation of China (Grant No.51905035), and the Science and Technology Innovation Special Zone Youth Project (Grant No. 2016312ZT00200203).

Institutional Review Board Statement: The study was conducted according to the guidelines of the Declaration of Helsinki, and approved by the Ethics Committee of Beijing Sport University (protocol code: 2019007H and date of approval: 2019.1.22).

Informed Consent Statement: Informed consent was obtained from all subjects involved in the study.

Data Availability Statement: The data presented in this study are available on request from the corresponding author. The data are not publicly available due to the data also forms part of an ongoing study.

Acknowledgments: The authors would like to thank all the participants for their cooperation.

Conflicts of Interest: The authors declare no conflict of interest. 


\section{References}

1. Jun-Young, J.; Wonho, H.; Hyundae, Y.; Hyunsub, P. A Neural Network-Based Gait Phase Classification Method Using Sensors Equipped on Lower Limb Exoskeleton Robots. Sensors 2015, 15, 27738-27759. [CrossRef] [PubMed]

2. Catalfamo, P.; Ghoussayni, S.; Ewins, D. Gait Event Detection on Level Ground and Incline Walking Using a Rate Gyroscope. Sensors 2010, 10, 5683-5702. [CrossRef] [PubMed]

3. Formento, P.; Acevedo, R.; Ghoussayni, S.; Ewins, D. Gait Event Detection during Stair Walking Using a Rate Gyroscope. Sensors 2014, 14, 5470-5485. [CrossRef]

4. Zhou, H.; Ji, N.; Samuel, O.W.; Cao, Y.; Zhao, Z.; Chen, S.; Li, G.; Sabatini, A.M. Towards Real-Time Detection of Gait Events on Different Terrains Using Time-Frequency Analysis and Peak Heuristics Algorithm. Sensors 2016, 16, 1634. [CrossRef]

5. Zhang, J.X.; Dou, S.F.; Su, H.L.; Cao, L. Variation characteristics of gait parameters during stair ascent and descent. J. Med. Biomech. 2016, 31, 266-271.

6. Dou, S.F. Biomechanics Charaderistic Research of Human Lower Limb during Walking on Stairs; Tianjin University of Science and Technology: Tianjin, China, 2016. (In Chinese)

7. Liu, Y.K. The Research of Gait Pattern and Gait Transition Based on the Motion Information; Northeastern University: Shenyang, China, 2014. (In Chinese)

8. Martin, G.; Julian, Z.; Florian, W.; Guoping, Z.; Sascha, L.; Martin, S.; Adrian, H. Lower limb joint biomechanics-based identification of gait transitions in between level walking and stair ambulation. PLoS ONE 2020, 15, e0239148.

9. Tae, H.J.; Sun, C.J.; Soo, B.S. The Spatio-temporal Analysis of Gait Characteristics during Ramp Ascent and Descent at Different Inclinations. J. Korean Phys. Ther. 2006, 18, 95-106.

10. Tae, H.J.; Hyun, K.S.; Soo, B.S. The 3-D Motion Analysis of Kinematic Variety on Lower Extremities during Ramp Descent at Different Inclinations. Phys. Ther. Korea 2006, 13, 16-25.

11. Leu, T.-H.; Li, J.-D.; Hong, S.-W.; Wang, T.-M.; Huang, S.-C.; Lu, T.-W. Trunk flexion strategy and the loads in the lower limbs when walking up surfaces of different slopes. Biomed. Eng. Appl. Basis Commun. 2012, 24, 295-305. [CrossRef]

12. Weiss, A.; Brozgol, M.; Giladi, N.; Hausdorff, J.M. Can a single lower trunk body-fixed sensor differentiate between level-walking and stair descent and ascent in older adults? Preliminary findings. Med. Eng. Phys. 2016, 38, 1146-1151. [CrossRef] [PubMed]

13. Yoav, G.; Raziel, R.; Hisham, R.; Amir, S.; Ronen, D.; Ilan, K.; Itshak, M. Age-related differences in pelvic and trunk motion and gait adaptability at different walking speeds. J. Electromyogr. Kinesiol. 2015, 25, 791-799.

14. Emmerik, R.E.A.v.; Wagenaar, R.C. Effects of walking velocity on relative phase dynamics in the trunk in human walking. J. Biomech. 1996, 29, 1175-1184. [CrossRef]

15. Gottschall, J.S.; Okorokov, D.Y.; Okita, N.; Stern, K.A. Walking Strategies during the Transition between Level and Hill Surfaces. J. Appl. Biomech. 2011, 27, 355-361. [CrossRef] [PubMed]

16. Zhang, S.; Pan, J.; Li, L. Non-linear changes of lower extremity kinetics prior to gait transition. J. Biomech. 2018, 77, 48-54. [CrossRef] [PubMed]

17. Sheehan, R.C.; Gottschall, J.S. Stair walking transitions are an anticipation of the next stride. J. Electromyogr. Kinesiol. 2011, 21, 533-541. [CrossRef]

18. Margareta, N. Basic Biomechanics of the Musculoskeletal System, 3rd ed.; LIPPINCOTT WILLIAMS \& WILKINS: Philadelphia, PA, USA, 2001; pp. 292-296. ISBN 9787117106023.

19. Riener, R.; Rabuffetti, M.; Frigo, C. Stair ascent and descent at different inclinations. Gait Posture 2002, 15, 32-44. [CrossRef]

20. Davis, R.B.; Ounpuu, S.; Tyburski, D.; Gage, J.R. A gait analysis data collection and reduction technique. Hum. Mov. Sci. 1991, 10, 575-587. [CrossRef]

21. Marigold, D.S.; Patla, A.E. Gaze fixation patterns for negotiating complex ground terrain. Neuroscience 2007, 144, $302-313$. [CrossRef]

22. Mentiplay, B.F.; Banky, M.; Clark, R.A.; Kahn, M.B.; Williams, G. Lower limb angular velocity during walking at various speeds. Gait Posture 2018, 65, 190-196. [CrossRef]

23. Liu, Y.; Lu, K.; Yan, S.; Sun, M.; Lester, D.K.; Zhang, K. Gait phase varies over velocities. Gait Posture 2014, 39, 756-760. [CrossRef] [PubMed]

24. Hebenstreit, F.; Leibold, A.; Krinner, S.; Welsch, G.; Lochmann, M.; Eskofier, B.M. Effect of walking speed on gait sub phase durations. Hum. Mov. Sci. 2015, 43, 118-124. [CrossRef]

25. Prentice Stephen, D.; Hasler Erika, N.; Groves Jennifer, J.; Frank James, S. Locomotor adaptations for changes in the slope of the walking surface. Gait Posture 2004, 20, 255-265. [CrossRef] [PubMed]

26. Peng, J.; Fey, N.P.; Kuiken, T.A.; Hargrove, L.J. Anticipatory kinematics and muscle activity preceding transitions from levelground walking to stair ascent and descent. J. Biomech. 2016, 49, 528-536. [CrossRef] [PubMed]

27. Chen, H.C.; Ashton-Miller, J.A.; Alexander, N.B.; Schultz, A.B. Stepping over obstacles: Gait patterns of healthy young and old adults. J. Gerontol. 1991, 46, M196-M203. [CrossRef] [PubMed]

28. Sheehan, R.C.; Gottschall, J.S. Ramp Angle, Not Plateau Height, Influences Transition Strategies. J. Appl. Biomech. 2016, 32, 449-453. [CrossRef] 